\title{
A Survey on Projector-Based PC Clustered Distributed-Rendering Large Screen Displays and Techniques
}

\author{
Munjae Song ${ }^{1}$ and Seongwon Park ${ }^{2}$ \\ ${ }^{1}$ Institute for Graphic Interfaces, 3rd Fl, Ewha-Sk Telecom Bldg. 11-1 \\ Daehyun-dong, Seodaemun-gu, Seoul 120-750, Korea \\ mjsong@igi.re.kr \\ ${ }^{2}$ Graduate School of Information, Yonsei University, 134 Sinchon-Dong, \\ Seodaemun-Gu, Seoul, Korea \\ seongwon@yonsei.ac.kr
}

\begin{abstract}
Large screen display systems are common display systems nowadays. Especially projector-based PC cluster large screen display systems share most of large screen display system market and they are main research topics today. A lot of researchers research on cluster algorithms, computation power improvements, high performance graphic rendering technologies, high speed buses, networks, and HCIs. Also, remarkable research results are being published by technical leading groups. Whereas, following groups who want research on large screen display have difficulties even to build a test system. Unfortunately, there are not enough information to build large screen display systems. In this paper, we survey on projector-based $\mathrm{PC}$ cluster large screen display technologies that use distributed rendering.
\end{abstract}

Keywords: Large screen display, Tiled display, PC cluster, Projectorbased, Distributed rendering.

\section{Introduction}

The projector-based PC cluster large screen display technology has a dramatic improvement a past decade. It has few advantages, and its advantages make PC clustered systems are popular. Next are the advantages of PC clustered large screen display systems.

- PC performance is improving very fast. Current CPUs have multiple cores and acceleration functions for vector calculation, e.g. MMX, 3D NOW and SIMD. Almost every PC is equipped with high-performance graphic cards having multiple rendering pipelines, programmable Shaders, and sometimes multiple GPUs.

- Using COTS (Commercial Off-The-Shelf) systems lowers system cost by cutting down prices and increasing product stability. Projector-based PC cluster large screen display systems which use COTS devices are widely used because they are low-price and reliable system. 
- There is a number of PC clustering open/free software which has abilities to customize and improve for building large screen display systems.

Nowadays, many researchers use seamless projector-based PC clustered distributed large screen display systems. We include our surveys, experiences of building large screen display systems. We believe this paper will help research groups who want build a projector-based PC clustered distributed large screen display system. Especially, we focus on research groups that research with large screen display systems, but do not want research on large screen display system deeply.

\section{Cluster Software}

In this section we survey cluster software, discriminating large screen display software. The cluster software has two types. One is image-based streaming software, another is distributed rendering software. Table 1 shows advantages and shortcomings of two types of large screen display software.

Table 1. Comparison table of large screen display software

\begin{tabular}{|l|l|l|}
\hline \multicolumn{1}{|c|}{ Type } & \multicolumn{1}{|c|}{ Advantages } & \multicolumn{1}{|c|}{ Shortcomings } \\
\hline $\begin{array}{l}\text { Image-based } \\
\text { streaming } \\
\text { software }\end{array}$ & $\begin{array}{l}\text { - Do not consider that update } \\
\text { scene graph, timer, random } \\
\text { number, and user input }\end{array}$ & $\begin{array}{l}\text { - Dedicate network devices for } \\
\text { wide bandwidth } \\
- \text { Clients do not use their } \\
\text { resource }\end{array}$ \\
\hline $\begin{array}{l}\text { Distributed } \\
\text { rendering } \\
\text { software }\end{array}$ & $\begin{array}{l}\text { - Typical network device } \\
\text { - Clients use their resource and } \\
\text { master has small loads } \\
-\begin{array}{l}\text { Handle big and complex data } \\
\text { with distributed system }\end{array}\end{array}$ & $\begin{array}{l}\text { - Difficulties of update scene } \\
\text { graph, timer, random number, } \\
\text { and user input }\end{array}$ \\
\hline
\end{tabular}

\subsection{Image Based Streaming Software}

There are a lot of image based streaming software, but in this section, check remarkable software. TeraVision [19], SAGE [8][23] and Juxta View[10] are remarkable research results.

\subsection{Distributed Rendering Software}

Following, we review remarkable distributed rendering software, some of them are open source and others are commercial products. Open source software are flexible but commercial products do not need additional development and are stable. Good classifications of distributed rendering software can be found in [27], [18]. Remarkable distributed rendering softwares are CAVE[15], VR juggler [3], Syzygy [24], Jinx [25], OpenSG [22], Chromium [6], NAVER [17], Nova [32][29]. 


\section{$3 \quad$ Hardware Devices}

Projector-based PC cluster large screen display system needs special hardware devices[33][7]. For example, projectors[2][4][16], graphic cards[14] [31], projector stages[28] [20] [30], and screens are needed for building large screen display systems.

\section{Software Requirements}

Some projector-based large screen displays need several features. These features are not essential functions but, make improve large screen display system's quality better. For example, edge-blending[12][11][5][13], NLDC (NLDC) [20][21][1][9] and Frame-lock. Fig. 1 shows implementation process with software features.

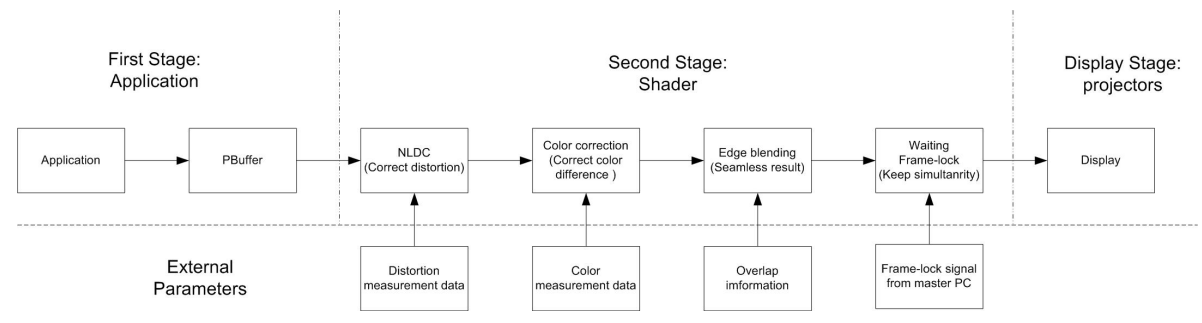

Fig. 1. Flow chart of implementation process

\section{Conclusion}

Large screen display systems are common display systems today. Especially projector-based PC cluster large screen displays are most popular large screen display systems. Projector-based systems are more useful for making seamless large screen display systems than multi-monitor systems. This paper shows a guide to build projector-based PC clustered large screen display systems. We believe this paper is useful to understand building projector-based large screen display systems.

Acknowledgments. This work was supported by the IT RD program of MIC/ IITA.[2005-S-604-02, Realistic Virtual Engineering Technology Development].

\section{References}

1. van Baar, J., Willwacher, T., Rao, S., Raskar, R.: Seamless Multi-Projector Display on Curved Screens. In: Deisinger, J., Kunz, A. (eds.) Eurographics Workshop on Virtual Environments (EGVE), pp. 281-286. ACM Press, New York (2003)

2. Barco: http://www.barco.com 
3. Bierbaum, A., Just, C., Hartling, P., Meinert, K., Baker, A., Cruz-Neira, C.: VR Juggler: A Virtual Platform for Virtual Reality Application Development. In: Proceedings of the Virtual Reality 2001 Conference, Yokohama, Japan, pp. 215-222. IEEE Computer Society, Los Alamitos (2001)

4. Christie Digital Systems, Inc., http://www.christiedigital.com

5. Hereld, M., Judson, I.R., Stevens, R.L.: Introduction to Building Projection-based Tiled Display Systems. IEEE Computer Graphics and Applications 20, 22-28 (2000)

6. Humphreys, G., Houston, M., Ng, R., Frank, R., Ahern, S., Kirchner, P.D., Klosowski, J.T.: Chromium: A Stream Processing Framework for Interactive Graphics on Clusters of Workstations. In: Proceedings of ACM SIGGRAPH 2002, San Antonio, Texas, pp. 693-702 (2002)

7. INFITEC GmbH: http://www.infitec.net

8. Jeong, B., Jagodic, R., Renambot, L., Singh, R., Johnson, A., Leigh, J.: Scalable graphics architecture for high-resolution displays. In: IEEE Information Visualization Workshop 2005, Minneapolis, MN, USA (2005)

9. Johnson, T., Gyarfas, F., Skarbez, R., Quirk, P.: Multi Projector Image Correction on the GPU. In: Poster presentation at Workshop on Edge Computing Using New Commodity Architectures (EDGE). Chapel Hill, North Carolina (2006)

10. Krishnaprasad, N.K., Vishwanath, V., Venkataraman, S., Rao, A.G., Renamhot, L., Leigh, J., Johnson, A.E.: Juxtaview: A tool for interactive visualization of large imagery on scalable tiled displays. In: 2004 IEEE International Conference on Cluster Computing, pp. 411-420. IEEE Computer Society Press, Los Alamitos (2004)

11. Li, K., Chen, Y.: Optical Blending for Multi projector Display Wall Systems. In: 12th Lasers and Electro-Optics Society 1999 Annual Meeting (LEOS '99), pp. 281282. IEEE Press, Los Alamitos (1999)

12. Majumder, A., Stevens, R.: Perceptual Photometric Seamlessness in Tiled Projection-Based Displays. ACM Transactions on Graphics 24, 118-139 (2005)

13. Mayer, T.: New Options and Considerations for Creating Enhanced Viewing Experiences. Computer Graphics 31, 32-34 (1997)

14. NVIDIA corporation: http://www.nvidia.com

15. Pape, D., Cruz-Neira, C., Czernuszenko, M.: CAVE Users Guide (1997), http://www.evl.uic.edu/pape/CAVE

16. Matsushita Electric Industrial Co. Ltd., http://panasonic.co.jp

17. Park, C., Ahn, S.C., Kwon, Y.-M., Kim, H.-G., Ko, H.: Gyeongju VR Theater: A Journey into the Breath of Sorabol. Presence 12, 125-139 (2003)

18. Raffin, B., Soares, L., Ni, T., Ball, R., Schmidt, G.S., Livingston, M.A., Staadt, O.G., May, R.: PC clusters for virtual reality. In: IEEE Virtual Reality Conference(VR2006), pp. 215-222. IEEE Computer Society, Los Alamitos (2006)

19. Singh, R., Jeong, B., Renambot, L., Johnson, A., Leigh, J.: TeraVision: a Distributed, Scalable, High Resolution Graphics Streaming System. In: Cluster Computing, 2004 IEEE International Conference. IEEE CNF, pp. 391-400 (2004)

20. Raskar, R., Browny, M.S., Yang, R., Chen, W.-C., Welch, G., Towles, H., Sealesy, B., Fuchs, H.: Multi-projector displays using camera-based registration. In: IEEE Visualization, IEEE CNF, San Fransisco, CA, USA, pp. 161-522 (1999)

21. Raskar, R., van Baar, J.: Low-Cost Multi-Projector Curved Screen Displays. In: International Symposium Scociety for Information Display (SID) (2005)

22. Reiners, D.: Opensg: A scene graph system for flexible and efficient reltime rendering for virtual and augmented reality applications. Dissertation, TU Darmstadt, Germany (2002) 
23. Renambot, L., Rao, A., Singh, R., Jeong, B., Krishnaprasad, N., Vishwanath, V., Chandrasekhar, V., Schwarz, N., Spale, A., Zhang, C., Goldman, G., Leigh, J., Johnson, A.: Sage: the scalable adaptive graphics environment. In: WACE 2004, Nice, France (2004)

24. Schaeffer, B., Goudeseune, C.: Syzygy: Native PC Cluster VR. In: IEEE VR Conference, Los Angeles, CA, USA, pp. 15-22. IEEE Computer Society, Los Alamitos (2003)

25. Soares, L.P., Zuffo, M.K.: Jinx: an X3D Browser for VR Immersive Simulation Based on Clusters of Commodity Computers. In: Ninth international conference on 3D Web technology, Monterey, California, USA, pp. 79-86. ACM Press, New York (2004)

26. SGI: http://www.sgi.com

27. Streit, A., Christie, R., Boud, A.: Understanding next-generation vr: classifying commodity clusters for immersive virtual reality. In: 2nd international conference on Computer graphics and interactive techniques in Australasia and South East Asia, pp. 222-229. ACM Press, New York (2004)

28. Surati, R.J.: A Scalable Self-Calibrating Technology for Seamless Large-Scale Displays. PhD thesis, Department of Electrical Engineering and Computer Sceince, Massachussetts Institute of Technology (1999)

29. VISIONMAX International LTD: http://www.visionmaxint.com

30. Li, Z., Varshney, A.: Calibrating Scalable Multi-projector Display Using Camera Holography Trees. In: Seventh Annual Symposium on Immersive Projection Technology (IPT 2002), Orlando, FL. IEEE Computer Society Press, Los Alamitos (2002)

31. 3Dlabs Inc., http://workstation.3dlabs.com

32. 3Digm Inc., http://www.3digm.com

33. 3D PERCEPTION: http://www.3d-perception.com/ 